\section{EBV covers its tracks}

Viruses that lie low inside cells after infection come under attack by the immune system when they reawaken and resume multiplying. Hislop et al. (page 1863) now reveal the mechanism used by the Epstein-Barr virus (EBV) to dodge host immunity during this reactivation phase.

EBV initially infects and replicates within oral epithelial cells but later quietly hides out in B cells. During this latent phase, EBV-infected B cells avoid the immune system by expressing very little viral antigen. To ensure viral spread and survival, however, EBV must reenter the replicative, or lytic, phase and invade new epithelial tissues.

Previous studies suggested that this herpesvirus also avoids the attention of the immune system during its reawakening. During the lytic phase, EBVinfected B cells dial down the activity of their transporters associated with antigen processing (TAPs) - transmembrane channel proteins that shuttle antigenic peptides into the ER, where they find their HLA partners. Infected $B$ cells thus display few viral antigens at the cell surface.

Known herpesviruses genes encoding TAP-inhibiting proteins were not found in the EBV genome. The authors therefore compared herpesvirus genomes to find a lytic phase TAP inhibitor gene in EBV and its closest relatives. Cloning and expression of candidate genes uncovered BNLF2a, which encodes a protein that blocked both the peptide-binding and ATP-binding sites on TAPs and thereby prevented it from translocating peptides into the ER.

This mechanism differs from those used by all other herpesvirus TAP inhibitors. The team is currently investigating how the relatively small BNLF2a protein blocks access to two distant sites on TAP. JEM

\section{All B cells need is BAFF}

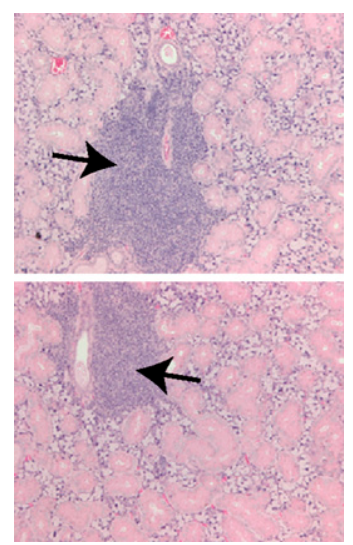

Lupus-inducing B cells (arrows) develop even when T cells are missing (bottom).
B cells usually conspire with T cells to break tolerance against the host and cause lupus. But Groom et al. (page 1959) now show that B cells don't always need T cells to egg them on. An activating cytokine empowers them to cause trouble all on their own.

This cytokine, called BAFF (B cellactivating factor), helps $B$ cells survive as they transit through developmental checkpoints. BAFF also enhances $T$ cell activation. With too much BAFF, however, even B cells that should have been eliminated-such as self-reactive ones-survive. The autoreactive antibodies first secreted by these cells are relatively harmless. But presumably with help from BAFF-activated T cells, these B cells switch their antibody genes and start producing pathogenic autoantibodies.

Groom et al. now find that the antibody-switching signal doesn't

have to come from T cells. Mice that overexpressed BAFF but lacked T cells still developed lupus. The B cells instead derived the extra push through their Toll-like receptors (TLRs) 7 and 9, whose expression was strongly enhanced by BAFF. The authors speculate that nucleic acids released by dying cells might trigger these TLRs on self-reactive $B$ cells.

The presence of disease-inducing antibodies even when T cells are absent might explain why not all lupus patients respond to treatments that suppress $T$ cell functions. The team is now investigating whether these patients have high levels of BAFF. JEM 